\title{
Blind Compensation of Linear Amplitude Distortions
}

\author{
Dario Fertonani, Alan Barbieri, Giulio Colavolpe, and Daniel Delaruelle
}

\begin{abstract}
The estimation and the compensation of particular amplitude distortions, typically affecting the consumer-grade receivers in broadband satellite communications, are considered. The distortion filter is modeled as a linear function of the frequency with unknown slope. We present a novel slope estimator, analytically derived by exploiting the spectral properties of the distorted signal, which results to be unbiased and does not exhibit false locks in the scenarios of practical interest. Since an ideal slope compensator would require an unmanageable filtering, we focus on a low-complexity compensation filter that ensures nearly the same performance. Extensive simulation results prove the effectiveness and the robustness of the proposed solution, which can also work blindly at very low values of the signal-to-noise ratio.
\end{abstract}

Index Terms-Blind compensation, broadband satellite communications, channel estimation, linear amplitude distortions.

\section{INTRODUCTION}

D ESPITE the large amount of literature dealing with the estimation and the compensation of unknown parameters [1], the future broadband satellite transmissions, as those based on the DVB-S2 standard [2], [3], raise new issues on the synchronization stage. One of the most critical problems is the presence of an amplitude distortion on the received signal, mainly due to the low noise block (LNB) and the coaxial cable at the consumer side [3]. After measurements on these devices, a model based on a linear filter whose amplitude response linearly depends, with unknown slope, on the frequency was proposed [4]. In conditions of broadband signaling, namely from 30 $\mathrm{MHz}$ ahead, the effect of the so-called slope distortion on the performance of the system is disruptive, and it must be properly estimated and compensated [4]. There exist various techniques for channel estimation that can cope with very general distortion models (see [5], [6] and references therein), but the general-purpose effectiveness of these solutions is paid in terms of complexity. In particular, when a very accurate estimation is required, the presence of a known-data training sequence is assumed [6]. In this paper, we address the problem of designing an ad-hoc compensation algorithm, characterized by a very limited complexity, that provides an accurate blind estimation, even at very low values of the signal-to-noise ratio.

Manuscript received February 14, 2007; revised May 21, 2008. First published June 27, 2008; last published August 20, 2008 (projected). This paper was presented in part at the 9th International Workshop on Signal Processing for Space Communications (SPSC 2006), ESTECNoordwijk, The Netherlands, September 2006.

D. Fertonani, A. Barbieri, and G. Colavolpe are with the Università di Parma, Dipartimento di Ingegneria dell'Informazione, Parma 43100, Italy (e-mail: fertonani@tlc.unipr.it; barbieri@tlc.unipr.it; giulio@unipr.it).

D. Delaruelle is with the Newtec Cy N. V., Sint-Niklaas 9100, Belgium (e-mail: daniel.delaruelle@newtec.be).

Digital Object Identifier 10.1109/TBC.2008.2000466
A novel fully-digital slope compensator is presented in both open-loop and closed-loop configurations [1]. The proposed estimation algorithm exploits the properties of the autocorrelation function of the received signal, and results computationally very simple. We describe a low-complexity compensation filter based on the first-order Taylor expansion of the ideal one, which should exhibit an hyperbolic frequency amplitude response and thus is impractical from a complexity viewpoint. We also considered higher-order approximations, but no significant improvement was found in scenarios of practical interest. The effectiveness of the proposed solutions is first proved by theoretical performance analyses, and then confirmed by extensive simulation results. In particular, the designed compensator results such robust that it can work blindly at very low values of the signal-to-noise ratio.

The remainder of this paper is organized as follows. In Section II, we describe the basic system model. In Section III, the proposed compensator is presented in both open-loop and closed-loop configurations. In Section IV, it is shown how to modify the compensator in order to make it effective in typical satellite communication scenarios. In Section V, some simulation results showing the performance of the proposed solutions are reported. Finally, Section VI gives some concluding remarks.

\section{SySTEM MODEL}

Let $x(t)$ be a zero-mean complex-valued signal with limited single-sided bandwidth $B_{x}$, and let us suppose that $x(t)$ is distorted into $y(t)$ by the slope filter

$$
H_{S}(f)=1+\delta f, \quad f \in\left[-B_{x}, B_{x}\right]
$$

where $\delta$ is an unknown deterministic parameter ranging in the interval $\left[-\delta_{\mathrm{MAX}}, \delta_{\mathrm{MAX}}\right]$. We will often exploit the time-domain interpretation of (1), according to which the slope filter produces a replica of the input signal plus a scaled version of its time derivative. In all scenarios of practical interest, the parameters $B_{x}$ and $\delta_{\mathrm{MAX}}$ are such that

$$
0 \leq \delta_{\text {MAX }} \ll \frac{1}{B_{x}} .
$$

To derive the basic slope compensator, we also assume that the autocorrelation function $R_{x}(\tau)$ of the input signal $x(t)$ is realvalued - we will explain how to cope with more general models in Section IV. The aim of this work is to design a fully-digital slope compensator working on the samples $y_{k}=y\left(k T_{s}\right)$, under the assumption that the sampling interval $T_{s}$ satisfies the Nyquist condition $B_{x} \leq 1 /\left(2 T_{s}\right)$ [7]. 


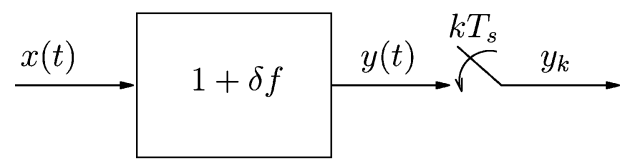

Fig. 1. Basic block diagram.

Referring to the block diagram shown in Fig. 1, the functions $S_{x}(f)$ and $S_{y}(f)$, respectively the power spectral density (PSD) of $x(t)$ and $y(t)$, satisfy the equation

$$
\begin{aligned}
S_{y}(f)= & S_{x}(f)\left[1+2 \delta f+\delta^{2} f^{2}\right] \\
= & S_{x}(f)-j \frac{\delta}{\pi}\left[(j 2 \pi f) S_{x}(f)\right] \\
& -\frac{\delta^{2}}{4 \pi^{2}}\left[(j 2 \pi f)^{2} S_{x}(f)\right] .
\end{aligned}
$$

Hence, applying the inverse Fourier transform to (3), we can write the following relationship between the corresponding autocorrelation functions

$$
R_{y}(\tau)=R_{x}(\tau)-j \frac{\delta}{\pi} R_{x}^{\prime}(\tau)-\frac{\delta^{2}}{4 \pi^{2}} R_{x}^{\prime \prime}(\tau)
$$

where $R_{x}^{\prime}(\tau)$ and $R_{x}^{\prime \prime}(\tau)$ are the first and the second derivative of $R_{x}(\tau)$ with respect to $\tau$. Thanks to the hypothesis of real-valued $R_{x}(\tau)$ and provided that $R_{x}^{\prime}(\tau) \neq 0$, taking the imaginary part of (4), we can write

$$
\delta=-\frac{\pi}{R_{x}^{\prime}(\tau)} \operatorname{Im}\left\{R_{y}(\tau)\right\}
$$

Since we are interested in fully-digital compensators working on the samples $y_{k}$, let us consider $\tau=n T_{s}$ (where $n$ can be any positive integer such that $R_{x}^{\prime}\left(n T_{s}\right) \neq 0$ ), so that the following equality results

$$
\begin{aligned}
\delta & =-\frac{\pi}{R_{x}^{\prime}\left(n T_{s}\right)} \operatorname{Im}\left\{R_{y}\left(n T_{s}\right)\right\} \\
& =-\frac{\pi}{R_{x}^{\prime}\left(n T_{s}\right)} \operatorname{Im}\left\{E\left\{y_{k} y_{k-n}^{*}\right\}\right\} .
\end{aligned}
$$

In Section III, we will exploit (6) to derive an estimator of the parameter $\delta$, which will be presented in both open-loop and closed-loop configurations.

\section{SLOPE COMPENSATION}

\section{A. Open-Loop Compensation}

If the statistical average $E\left\{y_{k} y_{k-n}^{*}\right\}$ in (6) is replaced by a temporal mean over a set of $L$ realizations, the following openloop estimator results

$$
\begin{aligned}
\hat{\delta} & =-\frac{\pi}{R_{x}^{\prime}\left(n T_{s}\right)} \operatorname{Im}\left\{\frac{\sum_{k=1}^{L} y_{k} y_{k-n}^{*}}{L}\right\} \\
& =-\frac{\pi}{L R_{x}^{\prime}\left(n T_{s}\right)} \sum_{k=1}^{L} \operatorname{Im}\left\{y_{k} y_{k-n}^{*}\right\} .
\end{aligned}
$$

It is easy to prove that the estimator (7) is unbiased, that is $E\{\hat{\delta}\}=\delta$, under the assumption of zero-mean input signal [8]. A classical figure for evaluating the effectiveness of an unbiased estimator is the mean squared error (MSE) of the estimate $E\left\{(\delta-\hat{\delta})^{2}\right\}$. Unfortunately, a simple closed form of the MSE for the estimator (7) does not exist, but a significant example where it can be exactly computed is reported in Section V. A comparison with the Cramer-Rao bound (CRB) [1] is also given, showing that the performance of the proposed estimator is very close to the theoretical limit.

Once achieved an estimate $\hat{\delta}$ of the value of the slope parameter, the distortion has ideally to be compensated by means of a filter exhibiting the transfer function ${ }^{1}$

$$
H_{C_{0}}(f)=\frac{1}{1+\hat{\delta} f}=\sum_{i=0}^{\infty}(-\hat{\delta} f)^{i}, \quad f \in\left[-B_{x}, B_{x}\right]
$$

the last equality holding provided that $|\hat{\delta}| B_{x}<1$, which is a condition assured by the hypothesis (2). Compensation filters of manageable complexity can be obtained by truncating the summation in (8): although higher-order approximations could in general be considered, we will focus on the first-order approximation

$$
H_{C}(f)=1-\hat{\delta} f, \quad f \in\left[-B_{x}, B_{x}\right]
$$

since, as shown in Section V and as expected under the hypothesis (2), it suffices for providing an excellent performance when values of $B_{x}$ and $\delta_{\mathrm{MAX}}$ of practical interest are considered. The complexity of the compensation filter (9) is particularly low: only one adder, one multiplier, and one derivative filter (scaled by a factor $j 2 \pi$ ) are required. The design of numerical differentiators is a widely-studied topic and it is known that the best solution consists of a FIR filter [9]. We consider a discrete-time 10th-order FIR filter (implying a 5-sample group delay), which provides an excellent accuracy in approximating the ideal derivative filter over a bandwidth of about $371 \%$ of the sampling rate, as shown in Fig. 2. Hence, the sampling rate must be designed by taking into account that the bandwidth over which the FIR filter approximates the ideal derivative filter must be larger than $B_{x}$, that is

$$
B_{x}<0.37 \frac{1}{T_{s}}
$$

The condition (10) could be obviously relaxed by resorting to higher-order FIR differentiators, which approximate the transfer function of the ideal derivative filter over a larger bandwidth.

Let us point out that the estimator (7) requires to perfectly know the value of $R_{x}^{\prime}\left(n T_{s}\right)$ : an error in the value of $R_{x}^{\prime}\left(n T_{s}\right)$ causes a proportional bias in the estimate $\hat{\delta}$. Since it is often difficult to get a spectral characterization of $x(t)$ as detailed as required to achieve a reliable estimation of the value of $R_{x}^{\prime}\left(n T_{s}\right)$, the relevance of this open-loop compensator is mainly theoretical. This drawback can be solved by resorting to the closed-loop compensator described in Section III-B.

\footnotetext{
${ }^{1}$ To keep the notation simpler, the frequency responses of the filters are written as if we were working in the continuous-time domain.
} 


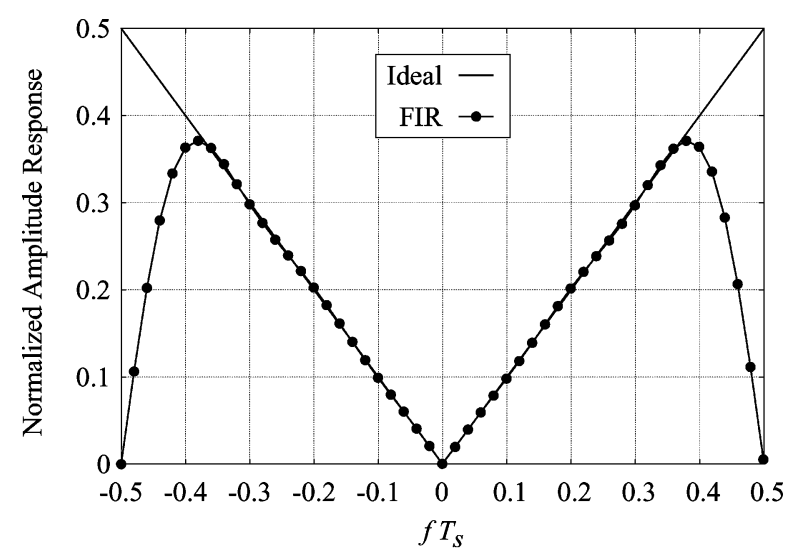

Fig. 2. Normalized amplitude responses of the ideal differentiator and of the considered 10th-order FIR filter.

\section{B. Closed-Loop Compensation}

Let us consider the block diagram reported in Fig. 3, and let $\hat{\delta}_{k}$, that is the estimate of $\delta$ at the sampling epoch $k$, be updated according to the classical closed-loop recursion [1]

$$
\hat{\delta}_{k+1}=\hat{\delta}_{k}+\gamma e_{k} \quad \text { with } \hat{\delta}_{0}=0
$$

where $\gamma$ is the step size of the loop and $e_{k}$ is a properly defined error signal. ${ }^{2}$ The crucial point of the algorithm is the design of the slope error detector (SED), that is the generation of $e_{k}$ in (11), such that the residual error $\Delta_{k}=\delta-\hat{\delta}_{k}$ vanishes on average for increasing values of $k$. Our aim is to design a SED such that

$$
E\left\{e_{k} \mid \Delta_{k}\right\} \propto \Delta_{k}
$$

since it is known [1] that, in the range over which it is satisfied, this condition ensures the effectiveness of the closed-loop estimator.

By applying to the scheme in Fig. 3 considerations similar to those leading to (6), it can be proved that

$$
\begin{aligned}
& \operatorname{Im}\left\{E\left\{z_{k} z_{k-n}^{*}\right\}\right\} \\
& =-\frac{\Delta_{k}}{\pi}\left[R_{x}^{\prime}\left(n T_{s}\right)+\frac{\delta\left(\delta-\Delta_{k}\right)}{4 \pi^{2}} R_{x}^{\prime \prime \prime}\left(n T_{s}\right)\right]
\end{aligned}
$$

where $\left\{z_{k}\right\}$ are the samples at the output of the compensator, and $R_{x}^{\prime \prime \prime}\left(n T_{s}\right)$ is the third-order derivative of $R_{x}(\tau)$ with respect to $\tau$, evaluated in $\tau=n T_{s}$. Hence, if we define

$$
e_{k}=A_{n} \operatorname{Im}\left\{z_{k} z_{k-n}^{*}\right\}
$$

\footnotetext{
${ }^{2}$ In Fig. 3 and in the discussion on the closed-loop compensator, we slightly abuse the notation by writing frequency responses involving the time-varying coefficient $\hat{\delta}_{k}$. Anyway, the step size $\gamma$ is assumed small enough that the estimates $\left\{\hat{\delta}_{k}\right\}$ result slowly varying and the frequency responses involving them are practically well defined.
}

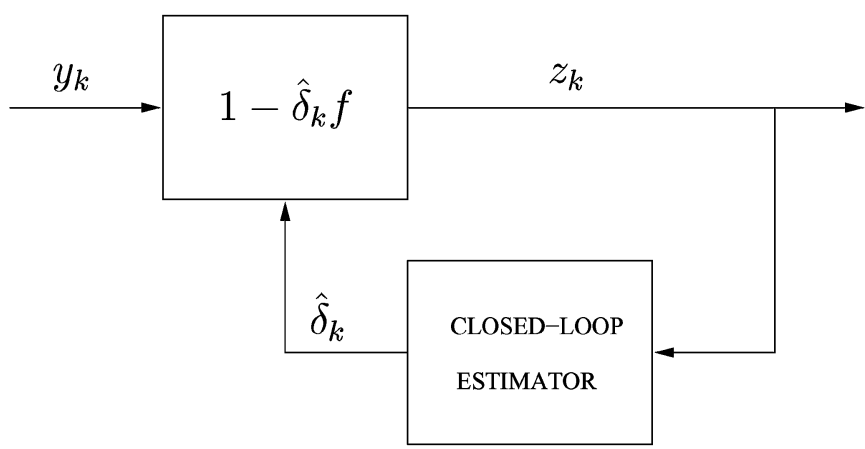

Fig. 3. Block diagram of the closed-loop compensator.

with $A_{n}=-\operatorname{sign}\left\{R_{x}^{\prime}\left(n T_{s}\right)\right\}$, the S-curve [1] turns out to be

$$
\begin{aligned}
E\left\{e_{k} \mid \Delta_{k}\right\}= & E\left\{A_{n} \operatorname{Im}\left\{z_{k} z_{k-n}^{*}\right\} \mid \Delta_{k}\right\} \\
= & \frac{\left|R_{x}^{\prime}\left(n T_{s}\right)\right|}{\pi} \Delta_{k} \\
& -\frac{\delta\left(\delta-\Delta_{k}\right) R_{x}^{\prime \prime \prime}\left(n T_{s}\right) A_{n}}{4 \pi^{3}} \Delta_{k}
\end{aligned}
$$

In the region of practical interest, the latter term in (15) is by far negligible with respect to the former, thus the obtained S-curve satisfies the target equation (12), proving that the described closed-loop compensator is unbiased and that no false locks can occur. Let us remark that this closed-loop estimator only requires to know the sign of $R_{x}^{\prime}\left(n T_{s}\right)$, instead of its exact value as required by the open-loop estimator (7), thus resulting easy to be implemented even in the case of partial knowledge on the spectral properties of $x(t)$.

\section{ApPliCATION to SATEllite COMMUNiCATIONS}

Let us now consider a satellite communication system employing linearly modulated digital transmissions. The complex envelope of the received signal, before being distorted by the slope filter (1), can be written as [5]

$$
r(t)=e^{j(2 \pi \nu t+\theta)} \sum_{i} c_{i} p(t-i T-\tau)+w(t)
$$

where $\left\{c_{i}\right\}$ are complex-valued modulation symbols, $p(t)$ is the equivalent shaping pulse at the receiver, $T$ is the signaling period, $\nu, \theta$, and $\tau$ are the frequency, phase, and timing offsets, and $w(t)$ is additive white Gaussian noise. We assume that $\left\{c_{i}\right\}$ are zero-mean and independent, and that $p(t)$ is the inverse Fourier transform of a root-raised-cosine function $P(f)$ with known roll-off parameter $\alpha$ [5]. Moreover, the unknown offsets $\nu, \theta$, and $\tau$ are assumed constant.

The signal $r_{s}(t)$, that is the received signal $r(t)$ after being distorted by the slope filter (1), is generally fed to an anti-alias filter (AAF) and sampled at a proper rate. The resulting samples are then processed by an automatic frequency control (AFC) loop, which has to perform a coarse non-data-aided frequency synchronization. The classical frequency-recovery algorithms exploit the spectral properties of the received signal [1], completely neglecting the slope distortion. Unfortunately, at large 


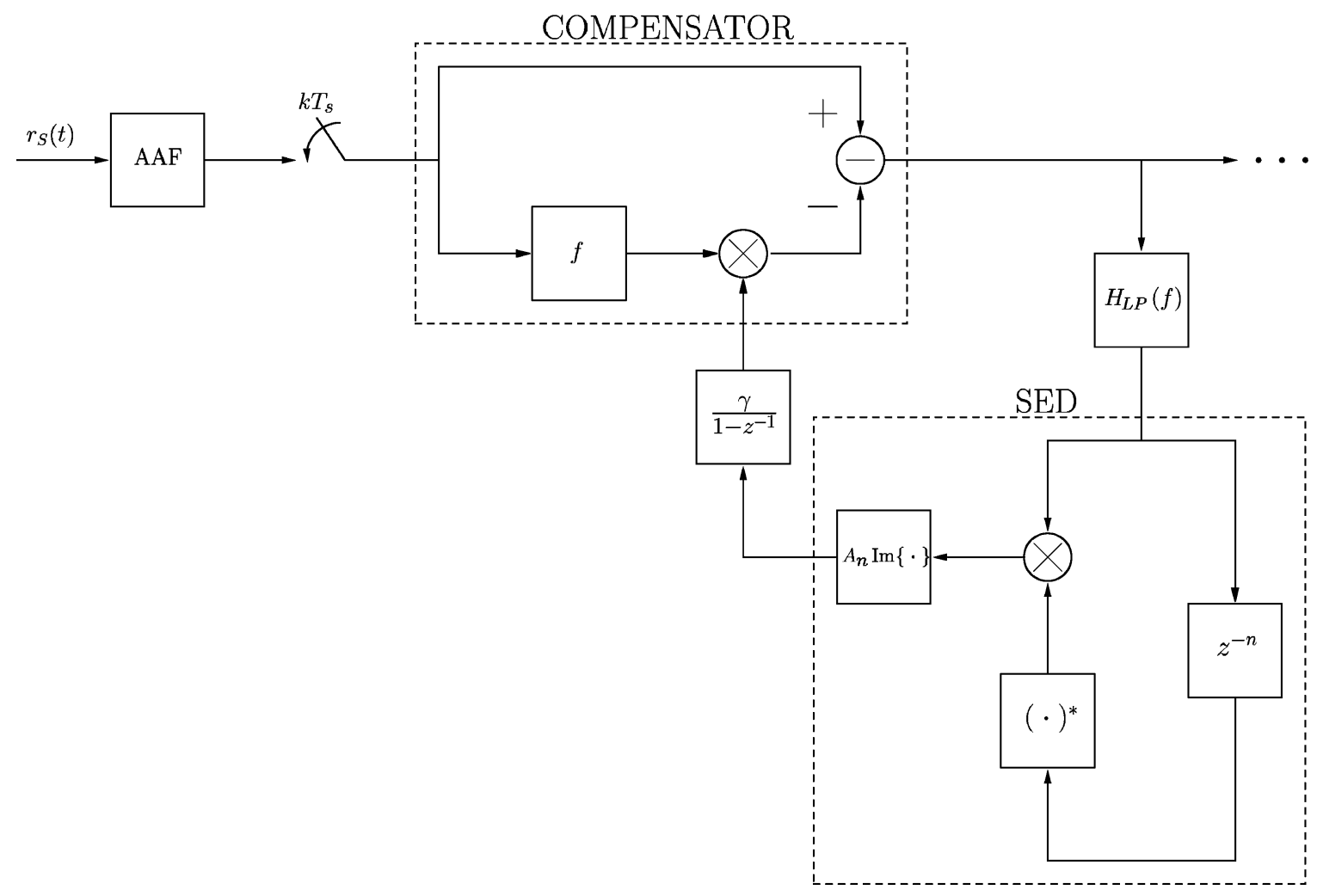

Fig. 4. Slope compensator for the system described in Section IV.

signaling bandwidths, namely from $30 \mathrm{MHz}$ ahead, the slope effect heavily alters such properties, thus causing large biases in the AFC loops and dramatically affecting the performance of the whole system [4]. In particular, such large biases cannot be tolerated by the timing-recovery algorithms typically implemented in satellite systems. Hence, a proper slope compensator must be placed before the AFC loop.

It is known [5] that the PSD $S_{r}(f)$ of the signal (16) satisfies the equation

$$
S_{r}(f) \propto|P(f-\nu)|^{2}+C
$$

where $C$ is a positive constant depending on the signal-to-noise ratio. It is worth to point out that the function $S_{r}(f)$ has even symmetry only if $\nu=0$, whereas the proposed slope compensator was derived in the hypothesis of an input signal which, before being distorted, has a real-valued autocorrelation or, equivalently, a PSD function with even symmetry. Hence, the compensator cannot work in the presence of a non-zero frequency offset, as it actually is since the compensator should be placed before the AFC loop. This problem can be solved by exploiting the fact that the function $P(f)$ is flat when $f$ is such that $|f|<$ $(1-\alpha) /(2 T)$. Let us fed $r(t)$ to an ideal rectangular low-pass filter $H_{L P}(f)$ with bandwidth $B_{L P}$, obtaining the output $x(t)$. If the condition

$$
B_{L P}<\frac{1-\alpha}{2 T}-|\nu|
$$

is satisfied, the PSD of $x(t)$ is such that

$$
S_{x}(f) \propto\left[|P(0)|^{2}+C\right] H_{L P}(f)
$$

and exhibits even symmetry, as required. This choice would ensure the effectiveness of the compensator, but it is not viable since the useful signal would be distorted by $H_{L P}(f)$. Hence, the final solution is to place the low-pass filter in the feedback path, as shown in Fig. 4, so that the filter $H_{L P}(f)$ does not affect the signal path. All these considerations still hold even if the ideal rectangular filter is replaced by any low-pass filter with even symmetry-in particular, we adopted a 5th-order Butterworth filter in the computer simulations. Let us point out that, when the system parameters are such that (18) results critical, the best solution is to place the closed-loop slope compensator inside the AFC loop, that is after the frequency compensator but before the frequency error detector [1], so that (18) can be satisfied after a transient stage.

\section{Simulation Results}

In this Section, the performance of the proposed slope compensators is assessed by means of computer simulations. To prove the robustness of the compensators, their behavior in the presence of the noise only is first reported. Then, a typical DVB-S2 scenario is considered.

Let complex white Gaussian noise with bandwidth $B$ input the distortion filter (1), and let us sample the distorted signal at the Nyquist rate. When the value of the slope parameter is $\delta=0$, it is possible to derive (by means of the standard method described in [10]) the closed-form expression of the CRB for $\hat{\delta}$, which turns out to be

$$
\frac{1}{2 K B^{2}}
$$




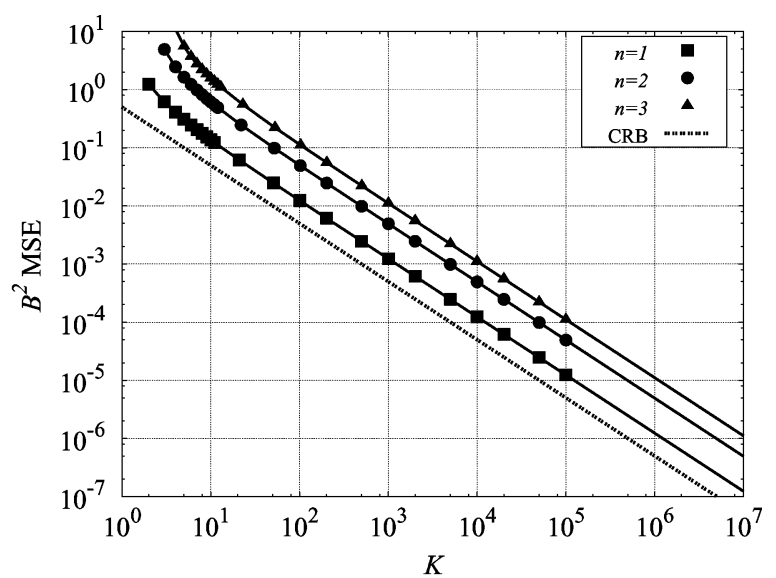

Fig. 5. Performance of the proposed open-loop compensator: the marks correspond to simulation results, while the lines correspond to the closed-form expression (21). The related CRB (20) is also reported as a benchmark.

where $K$ is the number of observed samples $\left\{y_{k}\right\}$, as well as that of the MSE for the open-loop estimator (7), which results

$$
\frac{\pi^{2} n^{2}}{8 L B^{2}}=\frac{\pi^{2} n^{2}}{8(K-n) B^{2}}, \quad K>n
$$

independently on the power of the noise (provided that it is non-zero) - we point out that the computation of (7) requires the observation of $K=L+n$ samples $\left\{y_{k}\right\}$. In Fig. 5 the CRB and the MSE, both normalized with respect to $1 / B^{2}$, are plotted versus the number of processed samples $K$. Some simulation results are also reported in Fig. 5, showing a perfect match of (21). Since, for large values of $K$, the MSE quadratically worsens with the value of $n$, the best choice is to set $n=1$. This choice ensures a degradation with respect to the CRB by a factor lower than 2.5 in terms of MSE for a given number of processed samples, or, equivalently, in terms of number of samples to be processed to achieve a given MSE.

Hereafter, the reported results refer to the closed-loop compensator described in Section III-B. In Fig. 6, the most important figures for evaluating the effectiveness of a closed-loop compensator, namely the acquisition time and the steady-state MSE, are reported, with the latter as a function of the former. Fig. 6 refers to the case of complex white Gaussian noise as input signal, limited to a bandwidth of $35 \mathrm{MHz}$ by means of a 5th-order Butterworth filter, and to a sampling rate equal to $100 \mathrm{MHz}$ according to (10). The slope parameter is set to $\delta=1 \mathrm{~ns}$, which is a typical value in DVB-S2 scenarios [4], and the acquisition time $T_{\text {acq }}$ is here defined as the number of samples to be processed, on average, to achieve an estimate $\hat{\delta}_{k}>0.999 \mathrm{~ns}$, starting from $\hat{\delta}_{0}=0$. The marks related to simulation results are reported together with interpolating functions enlightening the (approximately) linear relationship between the acquisition time and the MSE when both of them are plotted in logarithmic scale. For a given $n$, different marks refer to different values of the step size $\gamma$. As in the previous case, the curves do not vary with the power of the noise (provided that it is non-zero). Values of $n$ from 1 to 4 are considered, with the exception of $n=2$ since such a choice gives a very poor performance (in this case, $R_{x}^{\prime}\left(2 T_{s}\right) \approx 0$ ). The best performance is again obtained by setting $n=1$ : for a

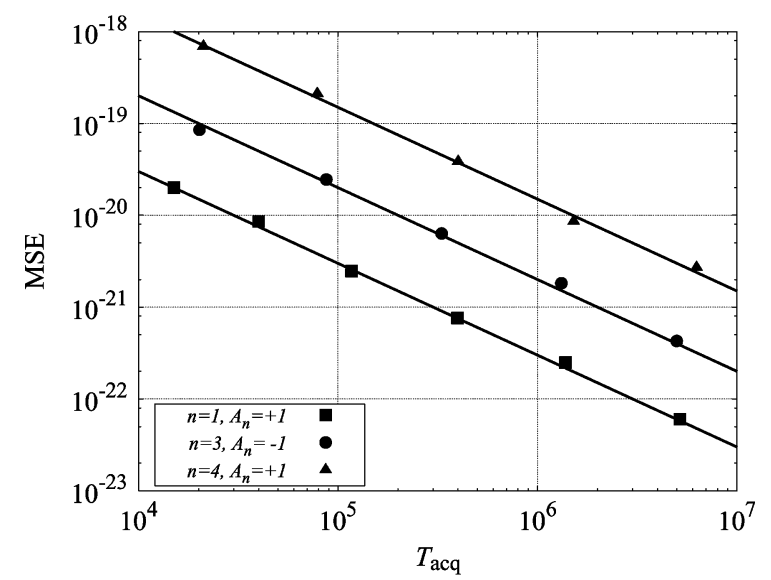

Fig. 6. Performance of the proposed close-loop compensator: the marks correspond to simulation results, while the lines are interpolating linear functions.

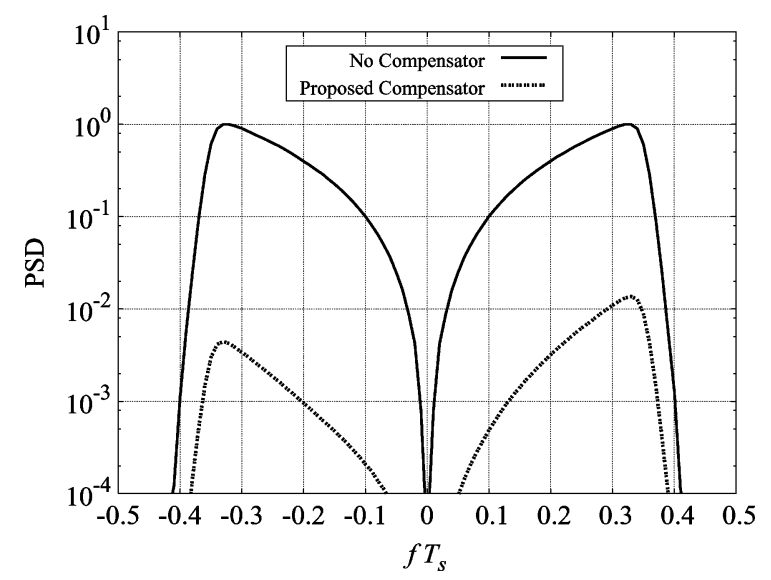

Fig. 7. Normalized power spectral density of the difference between the signal as it should be after an ideal slope compensator and the signal as it actually is in the absence/presence of the proposed closed-loop compensator.

given target MSE this solution is the fastest, or, equivalently, for a given target acquisition time this solution is the most accurate. Considering values of MSE lower than $10^{-21}$ as a target, ${ }^{3}$ a minimum of about $3 \cdot 10^{5}$ samples is thus to be processed even in the case of the best-performance curve-we notice that, when the symbol rate is of tens of Mbaud as in most DVB-S2 scenarios, the required acquisition time is in the order of few hundredths of second.

Let us finally consider a typical DVB-S2 scenario, characterized by an octal phase-shift keying (8-PSK) modulation, a signal-to-noise ratio of $5 \mathrm{~dB}$, a symbol rate of 45 Mbaud, a roll-off parameter $\alpha=0.3$, and a slope parameter $\delta=1 \mathrm{~ns}$. In such conditions, the slope distortion heavily alters the spectral properties of the received signal [4]. Hence, a significant measure of the effectiveness of a slope compensator in the steadystate condition is the PSD of the error due to the residual slope, that is the difference between the signal as it should be after an ideal compensator and the signal as it actually is. The results reported in Fig. 7, related to the closed-loop compensator

\footnotetext{
${ }^{3}$ This target results from simulations on the robustness of DVB-S2 systems not employing slope compensators [4]. It can be seen as the maximum tolerable value.
} 
whose block diagram is shown in Fig. 4, prove that this solution is noticeably effective in reducing both the peak values and the overall power of the error. In this case, the sampling rate is equal to $90 \mathrm{MHz}$, and the compensator is implemented by setting $n=1$ and $A_{n}=1$. It is clear that the proposed blind compensator practically recovers the original signal and, consequently, there is no need for complex data-aided equalization techniques to mitigate the residual slope distortion [4].

\section{CONCLUSIONS}

We have presented a novel compensator for amplitude distortions modeled as linear functions of the frequency. The compensator has been analytically derived by exploiting the spectral properties of the distorted signal, and its effectiveness has been proved by means of both theoretical analyses and simulation results. In particular, the designed compensator can work blindly at very low values of the signal-to-noise ratio. Hence, thanks to its performance and to its very low complexity, the proposed solution results much more convenient than the traditional general-purpose algorithms for channel equalization.

\section{REFERENCES}

[1] U. Mengali and A. N. D'Andrea, Synchronization Techniques for Digital Receivers (Applications of Communications Theory). New York: Plenum Press, 1997.

[2] ETSI, DVBS2 74r13, Digital Video Broadcasting (DVB): Second Generation Framing Structure, Channel Coding and Modulation Systems for Broadcasting, Interactive Services, News Gathering and Other Broadband Satellite Applications 2003.

[3] W. Fisher, Digital Video and Audio Broadcasting Technology, 2nd ed. Berlin Heidelberg: Springer Verlag, 2008.

[4] A. Barbieri, A. Cero, D. Fertonani, and G. Colavolpe, BSDT - Technical NOte TN01: Modem Algorithms Design Specification for the Broadband Forward Link With Linear Modulations - Part 1: Carrier Synchronization, July 2006, tech. rep., ESA Contract No. 19370.

[5] J. G. Proakis, Digital Communications, 4th ed. New York: McGrawHill, 2001.

[6] Z. Ding and Y. Li, Blind Equalization and Identification. New York: CRC, 2001

[7] H. Meyr, M. Oerder, and A. Polydoros, "On sampling rate, analog prefiltering, and sufficient statistics for digital receivers," IEEE Trans. Commun., vol. 42, pp. 3208-3214, Dec. 1994.

[8] A. Papoulis, Probability, Random Variables and Stochastic Processes. New York, NY: McGraw-Hill, 1991.

[9] A. V. Oppenheim and R. W. Schafer, Discrete-Time Signal Processing. Englewood Cliffs, New Jersey: Prentice-Hall, 1989.

[10] H. L. Van Trees, Detection, Estimation, and Modulation Theory - Part I. New York: John Wiley \& Sons, 1968. 